find a single measurement in the book: the effort has gone into capturing the shape of a bone or a tooth through its verbal dissection. In an era in which every morphological detail of a fossil can be captured using the most sophisticated technologies, and the information stored in digital archives, a book of anatomical descriptions may seem outdated. This is not so. Detailed morphological accounts are powerful analytical tools and rich sources of information, and this book represents an invaluable archive.

We are at a stage in human evolutionary studies when we are just starting to grasp the developmental mechanisms underlying specific morphologies. When new discoveries of the developmental biology of bone and teeth are made, we will be able to use this archive to document the evolution of a specific shape in our fossil relatives. This is a true thesaurus, in its literal meaning: a textbook to go back to several times, for different scopes.

Jacopo Moggi-Cecchi is at the Laboratori di Antropologia, Dipartimento di Biologia Animale e Genetica, Università di Firenze, 12 via del Proconsolo, 50122 Firenze, Italy.

\section{Winning the numbers game}

\section{The Honors Class: Hilbert's Problems and Their Solvers by Benjamin H. Yandell \\ A. K. Peters: 2002.496 pp. $£ 28, \$ 39$, 46 euros W. Timothy Gowers}

One of the best ways for a twentieth-century mathematician to achieve immortality was to solve a problem from the famous list of 23 compiled by David Hilbert and delivered to the International Congress of Mathematicians in 1900. The 'honours class' of Benjamin Yandell's title refers to those who managed this feat; his thoroughly researched and highly entertaining book tells us about their lives and about the problems themselves.

Yandell groups the problems according to their mathematical area, more or less as
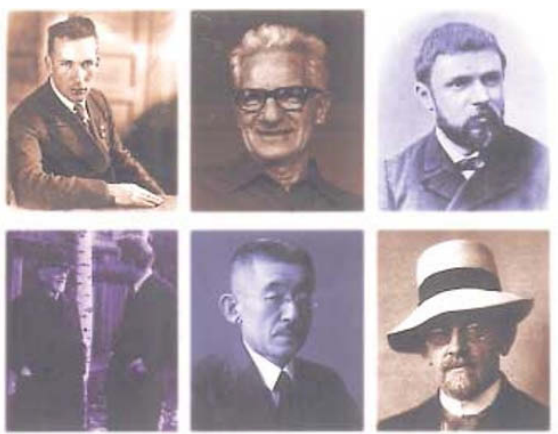

Sum people? Several mathematicians rose to the challenges set by David Hilbert (top row, centre).

\section{Marine masterpieces}

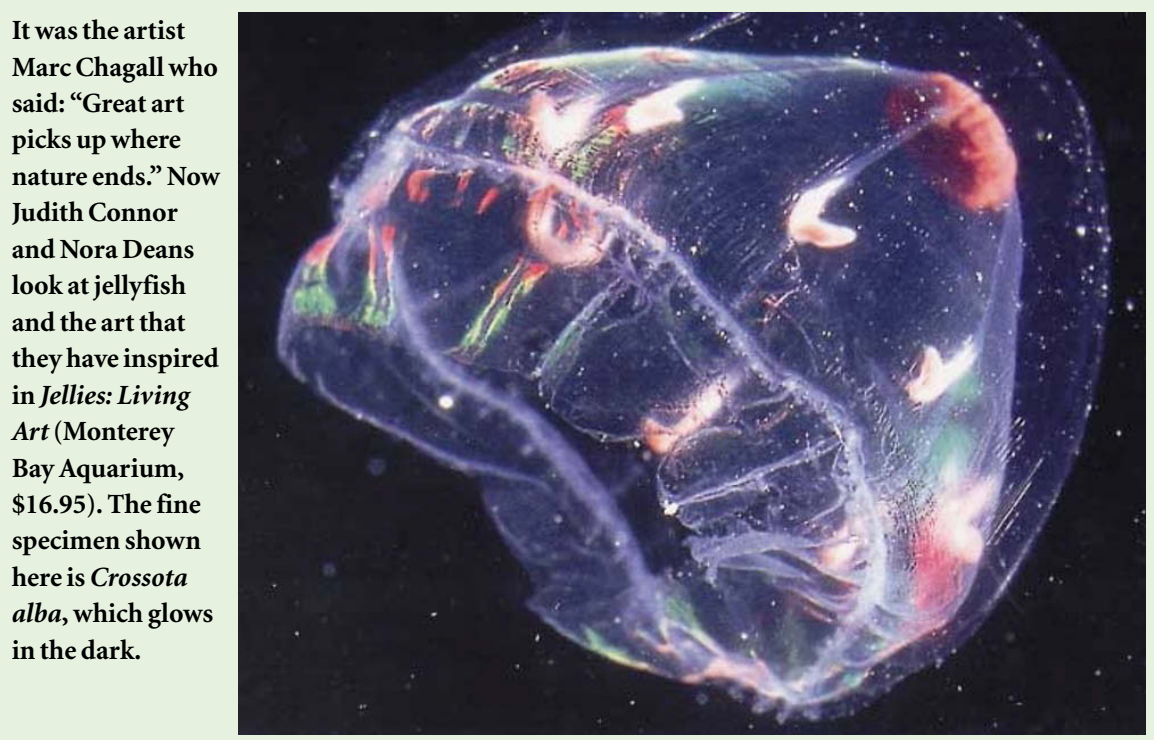

Hilbert did himself, although he reclassifies one or two problems because of the unexpected nature of their solutions. He then introduces each area, describes each problem within it, discusses the solution when there is one (by his reckoning only three remain unsolved), and intersperses the mathematics with plenty of biographical information about those who contributed to it. The result is a vivid history of much of twentieth-century mathematics from mathematical, personal and political perspectives - in Russia and Germany, at least, mathematics and politics were not entirely separate, with devastating results.

Occasionally one has too clear an idea of how the book was put together. Unlike many authors, Yandell does not try to hide behind his text. The book is is full of such phrases as "Michael [Artin] remarked to me that..." or "[Paul] Cohen thought... and I agree". In addition, some of the anecdotes, although amusing, are not really relevant. In the middle of a long discussion of A. N. Kolmogorov, we are treated to two stories about Sofya Kovalevskaya because she "throws an interesting light on Kolmogorov's early milieu". The remarkable story of Srinivasa Ramanujan is told because he wrongly claimed to have proved a result closely related to the Riemann hypothesis. And the general introduction to the section on number theory begins with a very short paragraph about G. H. Hardy before suddenly being interrupted by three well-worn tales about Paul Erdös. These are included because Erdös has "a connection to the eighth Hilbert problem", but we are never told what the connection is. (The eighth problem is the Riemann hypothesis again; it is one of the three that have not been solved.)

This is the second recent book on Hilbert's problems — the other, Jeremy Gray's The
Hilbert Challenge (Oxford University Press, 2000), was reviewed last year in Nature (410, 632-633; 2001). However, despite a necessary overlap between these books, they are very different in character and to some extent they complement each other. Gray's book was centred much more on the problems themselves - why they were chosen, why they were so influential, how even very differentlooking problems had similar underlying themes - whereas Yandell takes their importance largely for granted.

Gray included biographical details only when they help to explain the progress of the problems, whereas Yandell is far racier and revels in the human story. For example, you will not discover from Gray that Hilbert often became infatuated with pretty women, of whom Emmy Noether (possibly the greatest female mathematician ever) was not one, or that Pavel Aleksandrov liked to work outside under the burning sun wearing nothing but dark glasses and a Panama hat. Yandell's book is also significantly longer than Gray's, which allows him to discuss some of the mathematics in a more leisurely way. He does this well, although some of his discussions fall between two stools: unnecessary for the expert, but incomprehensible to the general reader.

Yandell turned his back on mathematics to become a writer after a distinguished undergraduate career at Stanford University. This makes him an ideal author for a book of this sort, to which he brings a rare combination of mathematical and literary sophistication. The Honors Class will be greatly enjoyed by anybody who wants to know more about mathematics and its practitioners.

W. Timothy Gowers is at the Centre for

Mathematical Sciences, Wilberforce Road,

Cambridge CB3 OWB, UK. 